\title{
Isolation and identification of an isomer of $\beta$-sitosterol by HPLC and GC-MS
}

\author{
Yi Sheng, Xiao-Bin Chen*
}

BS.Luh Food Safety Research Center, School of Agriculture and Biology, Shanghai Jiaotong University, Shanghai, China; xbchen@sjtu.edu.cn

Received 5 June 2009; revised 10 July 2009; accepted 12 July 2009.

\begin{abstract}
Phytosterols are a group of steroids alcohols which had been regarded as a functional factor. An unknown compound in phytosterol samples and phytosterol standard samples was detected by HPLC using symmetry $\mathrm{C}_{18}$ column. The quantity of the compound was increased with the enrichment of $\beta$-sitosterol. After being collected and analyzed by GC-MS and compared with standard diagram from Wiley and Nist standard chart library, it proved to be $\gamma$-sitosterol, a $24 \beta$ epimer of $\beta$-sitosterol.
\end{abstract}

Keywords: $\beta$-Sitosterol; $\gamma$-Sitosterol; Isomer; Stereochemistry; HPLC; GC-MS

\section{INTRODUCTION}

Plant sterols are steroid alcohols. Phytosterols regulate the membrane properties of the plant cells and participate in the control of membrane-associated metabolic processes. Sterols also play an important role in cellular and developmental processes in plants as precursors of brassinosteroids. They also act as substrates for a wide variety of secondary metabolites such as the glycoalkaloids, saponins. A major function of phytosterols in diet is the inhibition of absorption and subsequent compensatory stimulation of the synthesis of cholesterol. They are generally regarded as a kind of functional factor which could lower serum cholesterol and LDL-C level. Among different kinds of phytosterols, $\beta$-sitosterol has the most powerful serum cholesterol-lowering effect.

Phytosterols are made up of a tetracyclic cyclopenta $[\alpha]$ phenanthrene ring and a long flexible side chain at the $\mathrm{C}$ 17 carbon atom. The four rings $(\mathrm{A}, \mathrm{B}, \mathrm{C}, \mathrm{D}$, from left to right) have trans ring junctures, forming a flat $\alpha$ system. The most common representatives are sitosterol, stigmasterol and campesterol (4-desmethyl sterol). Campesterol and sitosterol have a $\triangle^{5}$ double bond and an additional one-carbon or two-carbon substituent in the side chain at C-24. Brassicasterol and stigmasterol have $\triangle^{5}$ and $\triangle^{22}$ double bonds, also an additional methyl or ethyl substituent at C-24 (Figure 1). These substituents are introduced by trans-methylation reactions. Methyl and ethyl substituents may have $\alpha$ or $\beta$ chirality. Most 24-ethyl sterols are $24 \alpha$-epimers, whereas 24-methyl sterols occur as mixtures of $24 \alpha$-epimers and $24 \beta$-epimers. Alkylation of C-24 is a reaction specific to plants.

Sitosterol is the principal sterol in plant materials. Generally it refers to $\beta$-sitosterol which has $\triangle^{5}$ double bond and $\alpha$-ethyl at C-24 [1]. The structure of $\beta$-sitosterol is definite. But little has been reported concerning $\gamma$-sitosterol, an epimer of $\beta$-sitosterol, which has been described as a $\triangle^{5}$ sterol and $\beta$-ethyl at C-24. Thompson (1963) [2] and Nishioka (1965) [3] respectively reported on $\gamma$-sitosterol in early years, but little chromatography information about how to separate these two epimers could be found.

In the course of an experiment aimed to enrich $\beta$-sitosterol, it was found that an unknown compound of small quantity also had been enriched. This compound had been neglected in the previous research and determination. It is important to define what this compound is.

\section{EXPERIMENTAL}

\subsection{Chemicals}

All chemical solvents are HPLC-grade, were purchased from Shanghai Chemical solvents company.

\subsection{Samples}

Four samples were used for determination by HPLC. Sample A was phytosterol raw material and its purity was $87.1 \%$. Sample B was a $\beta$-sitosterol enriched phytosterol product prepared in the lab. Sample C was a blended phytosterol standard sample (total phytosterols purity was 95.7\%) containing brassicasterol, stigmasterol, campesterol and $\beta$-sitosterol. Sample D was a stigmasterol standard sample with the purity of $97.3 \%$. All the standard samples were from Sigma. 


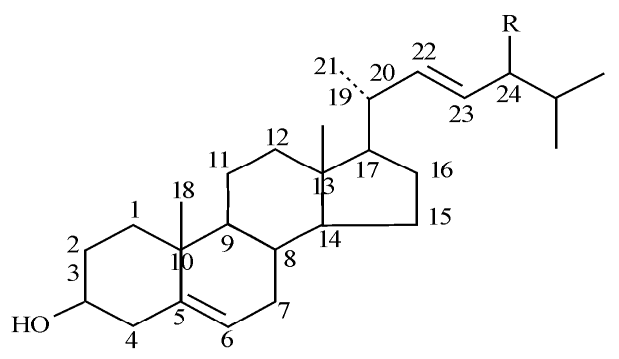<smiles>CCC(CCC(C)C)C(C)C</smiles><smiles>CC(C)C=CC(C)C(C)C</smiles><smiles>CC(C)CCC(C)C(C)C</smiles>

Figure 1. The structures of phytosterols.

\subsection{Sample Preparation}

The four samples were dissolved in absolute alcohol. The concentrations of sample A, B, C, and D were $0.7563 \mathrm{mg} / \mathrm{ml}, 0.8322 \mathrm{mg} / \mathrm{ml}, 1.360 \mathrm{mg} / \mathrm{ml}$ and $0.7520 \mathrm{mg} /$ $\mathrm{ml}$ respectively. Preparation of well-dissolved samples were achieved by ultra-sonic treatment. Undissolved particulate matter was removed by membrane filtration before the HPLC analysis.

\section{CHROMATOGRAPHY METHODS [4]}

\subsection{HPLC Conditions}

Waters 600 HPLC equipment combined with UV detector at UV $210 \mathrm{~nm}$. Symmetry $\mathrm{C}_{18}$ Column $(5 \mu \mathrm{m}, 3.9 \times 150 \mathrm{~mm})$ from Waters. Column temperature was $30^{\circ} \mathrm{C}$. Mobile phase: $\mathrm{MeOH}$ (HPLC grade). The flow rate was $1.0 \mathrm{ml} /$ min. Sample Loading was $30 \mu 1$.

\subsection{GC-MS Conditions}

TRAC-MS (Finnigan company), OV-1701 column (30m× $0.25 \mathrm{~mm} \times 0.25 \mu \mathrm{m})$ from Vertical Chromatography Co., Ltd. Mobile phase: He gas (99.99\% purity), flow speed was $0.8 \mathrm{ml} / \mathrm{min}$, split ratio was $10: 1$. Sample temperature: $280^{\circ} \mathrm{C}$. Column temperature: from $240^{\circ} \mathrm{C}$ and rose up to $265^{\circ} \mathrm{C}$ at the rising speed of $10^{\circ} \mathrm{C} / \mathrm{min}$. Remained at 265 ${ }^{\circ} \mathrm{C}$ for 40 minutes. Ionization mode: $\mathrm{EI}^{+}$. Electron energy: $70 \mathrm{eV}$. Interface temperature: $250^{\circ} \mathrm{C}$. Ion source temperature: $200^{\circ} \mathrm{C}$. Detection voltage: $350 \mathrm{~V}$. Sample loading: $0.5 \mu \mathrm{l}$.

\subsection{Separation of Four Samples by HPLC}

HPLC was applied to separate phytosterol samples for quantity determination. The RT (retention time) and quantity of each kind of sterol in phytosterol standard samples (sample C and D) were already known (Table 1). Sample B was prepared for the purpose of concentrating $\beta$-sitosterol. The unknown compound was also enriched while the $\beta$-sitosterol enriched. This phenomenon caused our interest. Usually the unknown compound we dis- cussed in this report was too little to be noticed, though it existed in almost all the samples. Their HPLC separation results are shown in Figures 2-5 (Sample A, B, C and D).

The information about the name and RT of each phytosterol in Figures 2-5 was listed in Table 1.

The first small peak is a compound with low concentration which was detected around $15 \mathrm{~min}$ to $15.3 \mathrm{~min}$ in sample A, B and C. It was a long chain alkane with SiOgroup. After collection and determination by GC-MS, this small compound was judged to be material lost from the $\mathrm{C}_{18}$ column.

The content of $\beta$-sitosterol in sample B was more than in sample A which was due to the enriching course of $\beta$ sitosterol. The content of $\beta$-sitosterol in raw material was $37.21 \%$ and in concentrated phytosterol sample was $56.46 \%$. Simultaneously, the unknown compound was also enriched in the experiment which showed the same concentrated tendency as $\beta$-sitosterol. Its content was raised from $4.92 \%$ in raw material to $6.55 \%$ in treated sample. It must be a kind of phytosterol for it has a similar chemical reaction ability and solvent dissolved property as $\beta$-sitosterol. This unknown compound was collected and analyzed by GC-MS for further study. Repeated collecting the unknown compound manually until $5 \mathrm{ml}$ was gathered when the unknown peak shown up from the HPLC UV detector. Dry the gathered sample by high purity of nitrogen gas, then dissolve it in $0.2 \mathrm{ml}$ cyclohexanone before GC-MS analysis.

\subsection{Analysis of the Unknown Compound by GC-MS}

The unknown compound was collected and analyzed by GC-MS. The information of MS was given from the Wiley and Nist standard chart library. The MS information is listed in Figure 6 and Figure 7.

The unknown compound (Figure 6) probably was an isomer of $\beta$-sitosterol. The name stigmast-5-en-3-ol was given by IUPAC (International Union of Pure and Applied Chemistry). The only difference from $\beta$-sitosterol was the stereochemistry in the position of C-24 ethyl. 


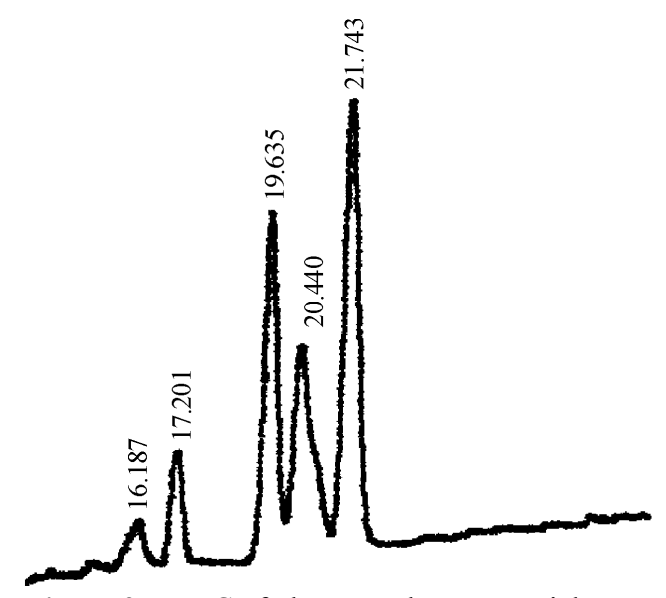

Figure 2. HPLC of phytosterol raw material.

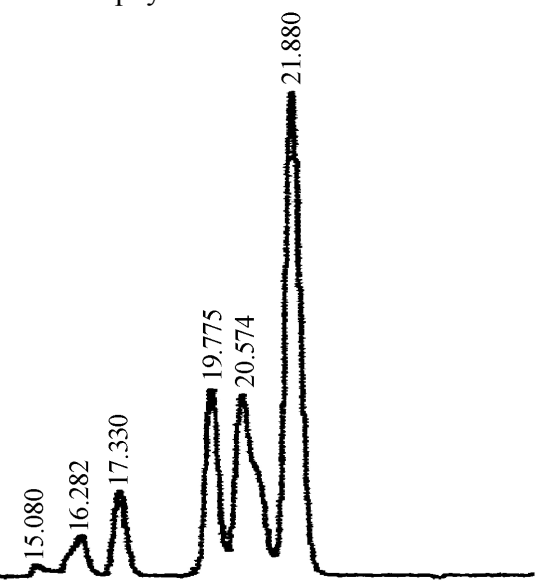

Figure 4. HPLC of $\beta$-sitosterol standard sample.

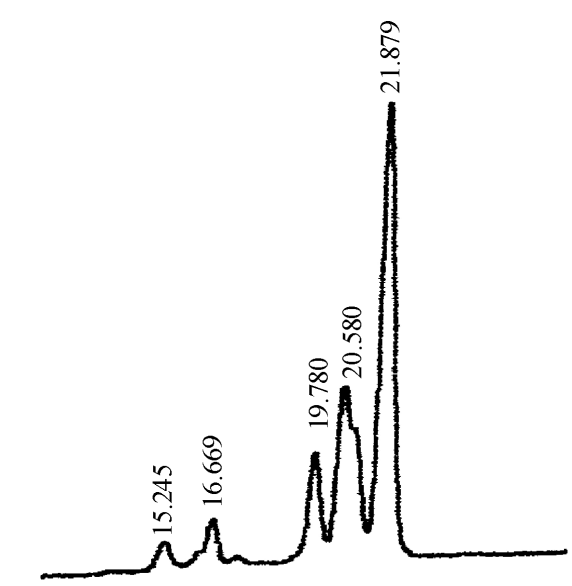

Figure 3. HPLC of phytosterol product.

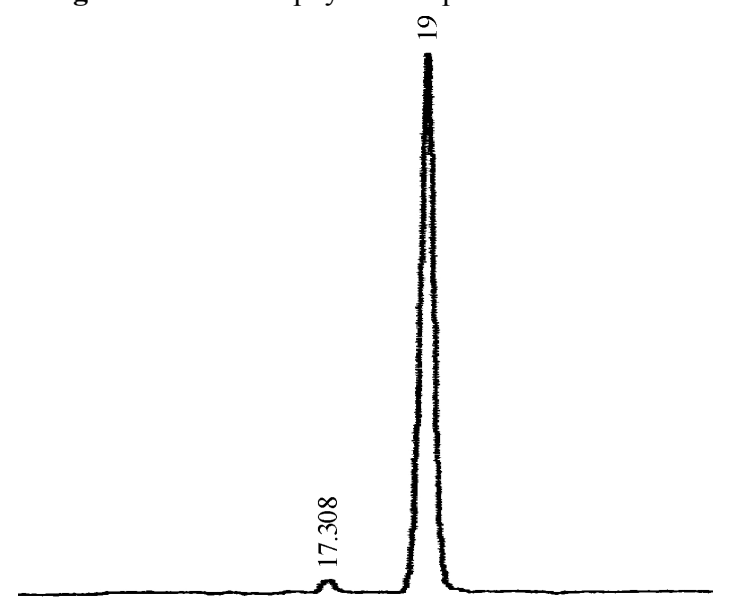

Figure 5. HPLC of stigmasterol standard sample.

Table 1. RT(min) of each sterol in sample A-D.

\begin{tabular}{rccccr}
\hline & \multirow{2}{*}{ Compound name } & Sample A & Sample B & Sample C & Sample D \\
RT(min) & RT(min) & - \\
\hline 1 & RT(min) & 15.245 & 15.080 & - \\
2 & Column loss & & 16.669 & 16.282 & 17.308 \\
3 & bnknown compound & 16.187 & 17.605 & 19.730 & 19.745 \\
4 & brassicasterol & 17.201 & 19.780 & - \\
5 & stigmasterol & 19.635 & 20.580 & 20.574 & - \\
6 & campesterol & 20.440 & 21.879 & 21.880 & - \\
\hline
\end{tabular}

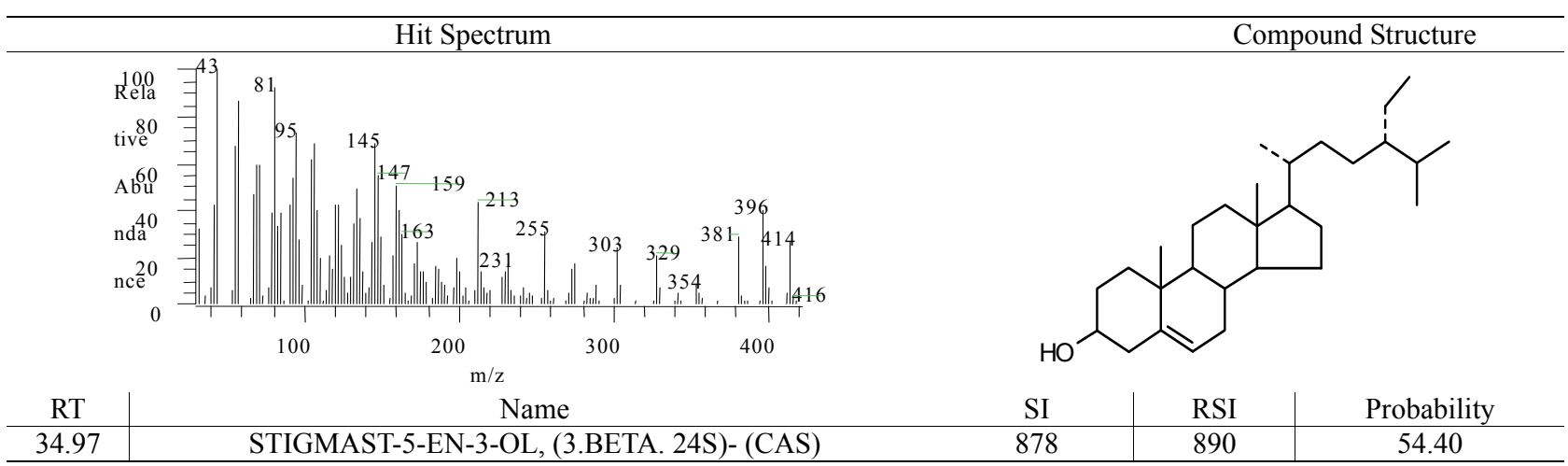

Figure 6. MS information of unknown compound. 


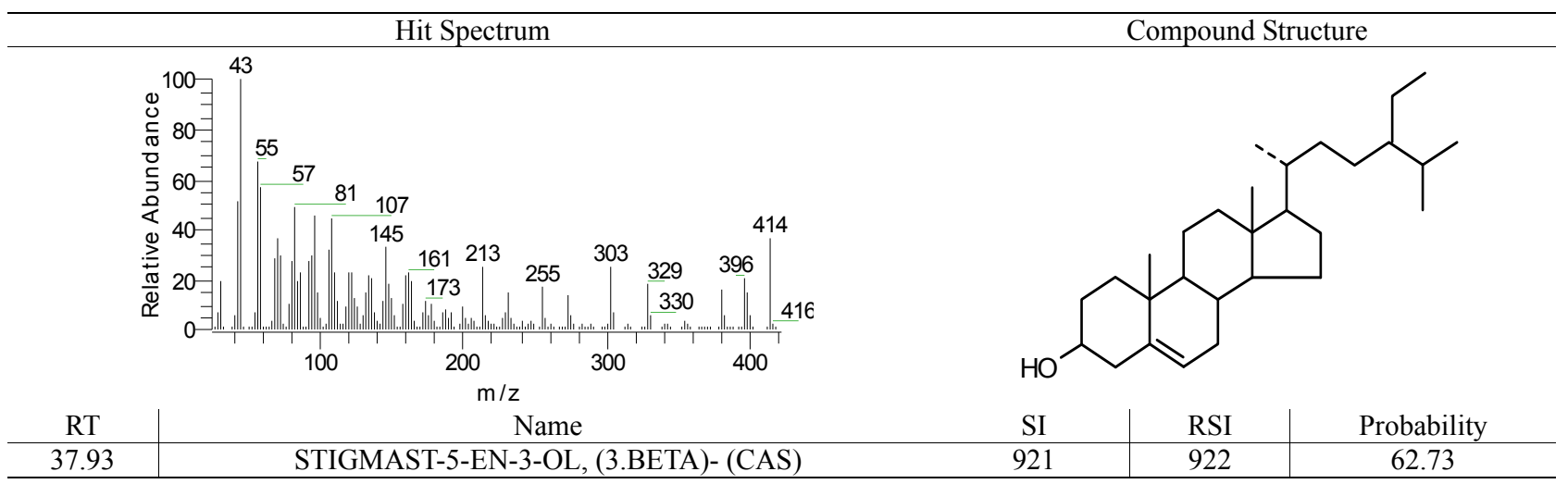

Figure 7. MS information of $\beta$-sitosterol.

\section{CONCLUSIONS}

$\beta$-sitosterol is one important kind of phytosterols that commonly occurs in raw material which was extract from deodorizer distillates. It is a $\triangle^{5}$ 4-desmethyl sterol with an additional ethyl substituent in the side chain at C-24. This 24-ethyl substituent has $\alpha$ chirality which had been already known. An unknown compound was found in the sample prepared in lab. The unknown compound could be separated from other phytosterols and collected by HPLC under our chromatography conditions. In further study, GC-MS information was given and shown that the unknown compound was probably to be an epimer of $\beta$ sitosterol, which was called $\gamma$-sitosterol by its trivial name. The only difference of these two epimers was the 24-ethyl substituent. The 24-ethyl in the side chain of $\gamma$-sitosterol has beta chirality, which was indicated as $24 \mathrm{~s}$ in the Wiley and Nist standard chart library.

It must be emphasized most strongly that the $\alpha-, \beta$ - assignments for side chain stereochemistry bear no relationship to the use of $\alpha$ - and $\beta$ - to define substituents attached to the sterol rings. The two systems of $\alpha / \beta$ assignments are quite unrelated.

Although $\gamma$-sitosterol is an important epimer of $\beta$-sitosterol, it has been neglected in past research. Especially the chromatography conditions for detecting and sepa- rating $\gamma$-sitosterol from other phytosterols in raw material samples. So, isolation and identification of $\gamma$-sitosterol by HPLC and GC-MS in this study provided a new, precise determination of phytosterol for further research.

\section{ACKNOWLEDGMENTS}

Thanks for the help from Professor Gu Wen-ying, Mr. Wang li-ping, Mr. Tao Guan-jun, Mr. Dai jun, they did a lot of work in chromatography. The project was sponsored by the funds of School of Agriculture and Biology, Shanghai Jiaotong University.

\section{REFERENCES}

[1] V. Piironen, D. G. Lindsay, T. A. Miettinen, etc. (2000) Plant sterols: Biosynthesis, biological function and their importance to human nutrition. J. Sci. of Food and Agric., 80, 939-966.

[2] M. J. Thompson, W. E. Robbins, G. L. Baker, etc. (1963) The nonhomogeneity of soybean-Gamma-Sitosterol. [J]. Steroids., 29(6), 407-418.

[3] I. Nishioka, N. Ikekawa, A. Yagi, ect. (1965) Studies on the plant sterols and triterpenes. [J]. Chem. Pharm. Bull., 11(5), 579-584.

[4] L. J. Goad and T. Akihisa, (1997) Analysis of sterol. Published by Blackie Academic \& Professional, London. 